\title{
Télématique : de la recherche sur les usages aux usages de la recherche
}

Telematics: From the Research About the Uses to the Uses of the Researches.

\section{Pierre Moeglin}

\section{(2) OpenEdition}

\section{Journals}

Édition électronique

URL : http://journals.openedition.org/edc/2790

DOI : $10.4000 /$ edc. 2790

ISSN : 2101-0366

Éditeur

Université Lille-3

Édition imprimée

Date de publication : 1 mai 1991

Pagination : 23-50

ISSN : $1270-6841$

Référence électronique

Pierre Moeglin, «Télématique : de la recherche sur les usages aux usages de la recherche », Études de communication [En ligne], 12 | 1991, mis en ligne le 12 janvier 2012, consulté le 19 avril 2019. URL

http://journals.openedition.org/edc/2790 ; DOI : 10.4000/edc.2790

Ce document a été généré automatiquement le 19 avril 2019

(c) Tous droits réservés 


\title{
Télématique : de la recherche sur les usages aux usages de la recherche
}

Telematics: From the Research About the Uses to the Uses of the Researches.

\author{
Pierre Moeglin
}

\section{Paradoxe initial.}

1 Les analyses qui vont suivre émanent d'un observateur peu impliqué dans le secteur proprement dit de la recherche sur la télématique. Si, de ce fait, elles bénéficient de l'avantage que confère la distance par rapport à l'objet étudié, elles sont en contrepartie soumises à l'inconvénient et aux limites d'une moindre familiarité avec les circonstances concrètes. C'est d'ailleurs la raison pour laquelle ces analyses visent davantage à soulever des interrogations concernant le statut et les conditions en général de la recherche en communication qu'à apporter des réponses aux problèmes spécifiques abordés par la recherche sur les usages de la télématique.

2 Si celle-ci, toutefois, fournit, en l'occurrence, matière à réflexion, c'est parce que les contradictions qu'elle traverse apparaissent à bien des égards symptomatiques de celles que connaissent, à des degrés divers, toutes les tentatives pour évaluer les innovations technologiques dans le champ de l'information et de la communication. Situation pour le moins surprenante, en effet, que celle de ses productions, simultanément stimulées et occultées, encouragées et mises aux oubliettes, et, semble-t-il, par ceux qui les ont réalisées autant que par ceux qui les ont commanditées.

3 D'où les questions que pose l'existence même de cette recherche, liées aux incertitudes de ses usages, en deçà de ses résultats sur les usages de la télématique : pour qui ? Pour quoi ?

4 Flagrant est en effet le décalage entre l'abondance des travaux sur les usages de la télématique et l'impression d'inutilité que donnent ces mêmes travaux : d'un côté, une 
mobilisation scientifique sans précédent ; de l'autre côté, l'absence de bilan officiel, une diffusion confidentielle, sans impact au moins apparent sur les choix politiques, sur les stratégies industrielles et a fortiori sur l'organisation d'un débat social autour de ces questions. Situation paradoxale par conséquent d'une recherche dont la raison d'être constitue en soi un problème.

\section{Une mobilisation sans précédent.}

Considérons pour commencer cette mobilisation, ses modalités et son ampleur. De tous les médias, le vidéotex est effectivement celui qui a le plus et le plus vite mis à contribution les chercheurs en communication. Ni la télévision - dont bien des aspects demeurent encore terrae incognitae - ni l'informatique, domestique ou professionnelle, ni le téléphone ou le câble ni les autres technologies dites «nouvelles » ne sont arrivées, sur des durées autrement plus longues, au résultat que la télématique, elle, a atteint en dix ans, avec la production d'un ensemble d'études possédant au moins trois caractéristiques notables.

Premièrement, elles s'alimentent à toutes les formes de recherche, faisant voisiner mesures quantitatives et observations cliniques, analyses descriptives et travaux normatifs, mises en perspective généalogiques et investigations prospectives, recherche développement et recherches critiques. La présence de ces dernières en particulier, et, plus généralement, de celles que leur dimension réflexive tend à faire sortir du cadre ordinaire (dans les milieux industriels) propre aux études appliquées, constitue à coup sûr un élément extrêmement original sur lequel nous reviendrons d'ici peu.

Deuxièmement, conséquence directe du point précédent, elles sollicitent tout l'éventail des disciplines relevant des sciences sociales, de la sociologie et de la psychologie jusqu'aux sciences politiques et à l'économie, en passant, entre autres, par l'ergonomie, la géographie et l'esthétique. En soi, cet élargissement du spectre disciplinaire est déjà remarquable, mais il l'est encore plus quand, rompant une seconde fois avec les pratiques ordinaires, certaines de ces études visent à développer des investigations transversales, mettant en perspective par exemple les aspects sociaux et les aspects économiques comme autant de niveaux d'analyse complémentaires.

8 Troisièmement, elles correspondent aux modalités contractuelles et aux contextes institutionnels les plus variés, depuis les enquêtes sur des points limités, réalisées à l'initiative de l'administration des PTT, des fournisseurs de services ou des autres acteurs industriels et politiques, jusqu'aux recherches indépendantes, à visée générale, conduites le plus souvent dans un cadre universitaire. La contribution d'équipes universitaires ou du CNRS, directement sollicitées ou s'intéressant spontanément au problème, n'est d'ailleurs pas l'élément le moins intéressant, comme on le verra, au sein des études sur la télématique.

9 Telles sont donc trois manifestations marquantes pour témoigner de ce qui fait le caractère exceptionnel de ces études : l'ampleur d'une mobilisation scientifique sans précédent sans doute, mais surtout et plus encore, derrière l'hétérogénéité des contributions ainsi sollicitées, la présence de disciplines et de recherches, réflexives et critiques, qui, pour la première fois, en tout cas de façon aussi systématique, sont associées au suivi et à l'évaluation d'un projet industriel. 


\section{Les usages sociaux : une problématique centrale.}

10 S'y ajoute un quatrième trait, qualitatif : même si ces études ne prennent pas toutes directement pour objet les usages de la télématique, elles s'y rapportent cependant peu ou prou, contribuant par cela même à instaurer et structurer un véritable champ de recherche par rapport auquel la notion d'usage et les problématiques qui en découlent se voient assigner une position centrale.

11 S'agissant en effet du vidéotex, technologie particulièrement ouverte, l'évolution des différentes utilisations auxquelles elle a su satisfaire au fur et à mesure - et que, dialectiquement, elle a suscitées - est devenue l'un des paramètres majeurs à prendre en compte. Cela tient essentiellement à ce que ce sont la diversité de ces utilisations et leur progressive extension qui ont permis à la télématique d'opérer sa conversion en passant du statut de simple innovation technique à celui de médium à part entière. Extension d'autant plus déterminante que ces utilisations se sont en effet considérablement modifiées et diversifiées tout au long de la décennie: débutant par la rudimentaire interrogation de l'annuaire électronique, telle qu'expérimentée en Ile et Vilaine à la fin des années 70, s'élargissant à la consultation d'un ensemble varié de bases de données, puis à la pratique d'autres services interactifs, notamment la messagerie, pour en arriver aujourd'hui aux applications en bureautique, monétique, gestion et vente par correspondance.

12 Du même coup et, pourrait-on dire, proportionnellement la réflexion théorique s'est ellemême développée et amplifiée, un peu comme si la multiplication et la diversification des utilisations constituaient la plus forte incitation à chercher à les penser dans un cadre plus intégré et plus élaboré. Ainsi voit-on en effet l'enrichissement des problématiques s'effectuer par déplacement du questionnement initial, qui donnait la priorité à l'analyse des utilisations - leurs modalités empiriques, leur volume, les obstacles à leur mise en oeuvre, les secteurs d'activité où elles s'inscrivent, etc. -, et par intégration de ce questionnement dans une problématique plus générale, celles des usages, qui devient alors l'un des centres de gravité de la recherche sur la télématique.

Cette problématique recouvre elle-même toute une série d'interrogations non plus seulement sur les emplois de la télématique mais, plus fondamentalement, sur les filiations et les génèses de ses usages, sur les logiques sociales qui en déterminent la formation et sur l'autonomie relative dont ils témoignent, au fur et à mesure de leurs évolutions, par rapport aux logiques régissant l'offre marchande. Par conséquent, l'on peut dire que, du questionnement sur les utilisations à la problématique sur les usages, la recherche sur la télématique tend à se structurer en adoptant un cadre d'analyse centré sur les stratégies des utilisateurs dans leurs interactions avec les stratégies des promoteurs industriels et commerciaux.

\section{Ce que cache l'effervescence.}

Le nombre et le volume de toutes ces productions scientifiques suffiraient à confirmer notre constat initial concernant la vitalité de ce thème et l'intérêt qu'il a suscité : plusieurs milliers de pages de rapports, d'articles, de livres et de thèses. La bibliographie sélective qui figure dans le numéro commun TIS et Réseaux sur « Dix ans de vidéotex » ne 
mentionne pas moins de 85 ouvrages, 102 rapports, 27 numéros de revue, sans oublier de multiples manifestations scientifiques et, pour célébrer la décennie, un important colloque organisé par le CNET où - fait exceptionnel- voisinèrent chercheurs en sciences sociales, décideurs et ingénieurs.

Ce qui, en revanche, peut, à certains égards, susciter la perplexité, c'est l'origine de cette abondance et les raisons d'une telle mobilisation. Par exemple, les signataires de l'éditorial du numéro conjoint TIS et Réseaux $(1989$, p. 9) s'interrogent sur cette situation en se demandant si cette " effervescence " ne cache pas une "sorte de fuite en avant", par rapport à laquelle a contrario la situation actuelle traduirait plutôt un certain essoufflement. L'idée de la fuite en avant caractérise en effet assez bien le processus d'accumulation des travaux entre 1981 et 1989. Mais elle décrit plus qu'elle n'explique. Ce dont, en fait, il conviendrait de rendre raison, c'est du mécanisme qui a provoqué cette accumulation, du côté des chercheurs tout autant que de celui de leurs commanditaires.

Quelques années auparavant, P. Flichy $(1984$, p. 1) relevait déjà le phénomène en faisant remarquer également que

parmi les nouvelles technologies de communication qui ont émergé ces dernières années, la télématique est incontestablement celle qui a fait naître le plus de littérature sociologique.

Et d'en proposer une explication:

La raison en est sans doute que l'État en tant que financeur de la recherche contractuelle oriente prioritairement les sociologues vers les systèmes techniques dont il assure le développement (ibidem).

S'ajoutant à la réponse précédente pour le préciser, celle-ci met explicitement l'accent sur les facteurs institutionnels, à travers l'influence de la commande publique sur une recherche sociale dépendant fortement des objectifs et des stratégies industrielles. Ce faisant, cette explication a le mérite de rompre avec la vision idéalisée d'une recherche sur les usages de la télématique qui serait née et se serait développée comme par génération spontanée. En réalité, dit en substance P. Flichy, cette recherche n'est importante que parce qu'elle l'est devenue. Et elle n'est devenue importante que parce que s'y attachent des enjeux financiers et, plus encore, politiques. Pourtant, du point de vue des usages et des pratiques sociales, ajouterons-nous, le magnétoscope a probablement une portée sans commune mesure avec celle de la télématique. Mais s'il n'a suscité aucune des investigations qu'a engendrées la télématique, c'est que les considérations financières n'y intervenaient pas de la même manière.

Restent néanmoins en suspens plusieurs questions déterminantes que l'explication précédente n'arrive pas à épuiser. Par exemple, pourquoi l'État a-t-il ainsi orienté les chercheurs sur la télématique comme il ne l'a fait pour aucune des autres technologies dont il est également le promoteur et dont le poids, du point de vue des investissements et des objectifs industriels, n'est pas moindre : câble, satellite, carte à mémoire ? Pourquoi, demanderons-nous également, l'État l'a-t-il fait par le biais des PTT, plaçant celles-ci dans la délicate position de juge et partie, au lieu de faire appel à d'autres instances, existantes ou à créer, qui auraient été moins engagées dans la promotion de Télétel et, du même coup, se seraient révélées mieux placées pour faire procéder à des évaluations objectives et pour en tirer les leçons nécessaires ? Pourquoi, enfin, cette effervescence, hier, et, aujourd'hui, son tarissement? Tarissement d'autant moins explicable a priori que le vidéotex est loin d'être sorti de la phase de haute surveillance et qu'au contraire les signes actuels de saturation du marché grand public ont de quoi inquiéter ses promoteurs. 
Toutes ces questions ont un point commun : elles tendent à introduire le soupçon par rapport à l'idée communément admise d'une recherche sociale naturellement et logiquement sollicitée par des promoteurs industriels et des décideurs politiques, confrontés à des environnements excessivement complexes et faisant du même coup appel à des experts capables d'éclairer leurs choix. Autrement dit, ces questions visent à suggérer que derrière le discours officiel - discours partagé (au moins pendant un certain temps) par les commanditaires et les commandités- et spécialement derrière le discours de l'expérimentation sociale qui en condense tout l'argumentaire, d'autres considérations interviennent peut-être. Un autre jeu se serait joué, auquel celui de la recherche et de l'expertise sur les usages aurait servi de paravent, parfois d'alibi, et auquel la télématique aurait fourni une sorte de prétexte. A la lumière de cette reformulation et sous réserve des nécessaires confirmations, l'interrogation initiale, celle des usages de la recherche sur les usages, changerait du tout au tout. Elle serait en somme de savoir quel est ce jeu et à quoi, dans ces circonstances, il peut avoir servi.

\section{Des recherches inutiles?}

21 S'il est hasardeux de chercher à identifier, à plus forte raison, d'essayer de mesurer pratiquement un phénomène aussi complexe que l'utilité des recherches sociales en télématique, serait-ce à l'aune de l'influence qu'elles ont eue ou non, l'on est quand même, sur des exemples précis, en droit de relever plusieurs éléments susceptibles d'illustrer le second terme de notre paradoxe initial : à partir du constat d'apparente étanchéité entre secteurs de la recherche et instances décisionnelles, l'impression de disproportion que donne le déploiement scientifique autour de la question des usages de la télématique par rapport à ce qu'est - et a été -la réalité de son impact.

N'est-ce pas par exemple un indice déjà assez significatif qu'aucune proposition émanant des chercheurs, pourtant si massivement mobilisés, ne soit venue alimenter des débats publics sur les enjeux sociaux et culturels de la télématique, à commencer par celui du début des années 80 - vite étouffé, au demeurant - que lança la presse écrite, le temps d'obtenir les garanties et privilèges qu'elle réclamait? Au contraire, lorsque, rétrospectivement, l'on examine les arguments échangés par les partisans et les adversaires de la télématique à l'époque, on est surpris du degré d'abstraction de leurs analyses et de l'impossibilité consécutive d'organiser la réflexion que, dès 1978, S. Nora et A. Minc appelaient pourtant de leurs voeux sur l'impact de "l'informatisation de la société ». A plus forte raison voit-on s'éloigner la perspective d'une authentique concertation sur les choix politiques et industriels présidant au lancement du Minitel.

Pêle mêle reviennent au contraire plus ou moins adaptés aux nouveaux contextes les vieux fantasmes de l'informatique des années 60 , depuis la référence orwellienne, sous sa version " Big brother " ou sous celle, édulcorée, du " grand chaudron » cher à Bruno Lussato, jusqu'aux utopies techno-démocratiques et téléconviviales dont l'un des pères, Ivan Illich, vint en personne et à l'invitation de la DOT, porter la caution au Télétel made in France.

De même l'excessive généralité des débats parlementaires sur Télétel, mis à part peutêtre ceux sur le thème " informatique et liberté » (mais justement importé du secteur de l'informatique), est assez révélatrice du peu d'influence de la recherche sur les instances politiques de décision et d'arbitrage. C'est que, sans prendre nécessairement pour idéal la 
pratique nord américaine du technological assessment, il aurait quand même pu se faire que des questions qui devaient justement être analysées et discutées dans les milieux de la recherche soient reprises en compte dans ces débats, comme de semblables l'ont d'ailleurs été, au moins partiellement, pour d'autres débats, dans les mêmes lieux, sur le câble ou la télévision.

Parmi ces questions, celles, par exemple, qu'au début de la décennie, plusieurs équipes d'évaluations soulevaient simultanément - avec des points de vue parfois divergents - sur les conditions de l'appropriation sociale de la télématique dans les contextes de l'information locale, en milieu rural ou par rapport aux transformations éventuelles des rapports entre administration et administrés, auraient pu fort à propos être réactivées et, faisant l'objet d'une discussion publique, introduire du même coup un salutaire contrepoint aux discours promotionnels et aux argumentaires corporatistes.

A l'inverse, n'est-il pas aussi significatif que l'interruption, réalisée brutalement ou en douceur, des chantiers expérimentaux de Vélizy, Strasbourg, Nantes, Grenoble et d'ailleurs n'ait été, sinon dénoncée, du moins regrettée publiquement par aucun parti ou personnalité politique ni par aucun mouvement social organisé ? A priori, l'on peut pourtant supposer que ces chantiers auraient fourni des données intéressantes, ne seraitce qu'en montrant par leur diversité même qu'il n'y a pas pour l'implantation de la télématique de one best way. Dès lors, leur suivi, réalisé par des structures extérieures travaillant pour les instances de décision, aurait été une occasion privilégiée pour ces deux ensembles de protagonistes de donner un contenu concret, à la fois institutionnel et scientifique, à l'idéal, récurrent mais flou en ce début des années 80 , de l'expérimentation sociale. Or, le moins que l'on puisse dire est que cette occasion a été manquée.

\section{L'expertise absente.}

Il serait tentant mais encore prématuré à ce stade de chercher à savoir si la responsabilité de ce qui, jusqu'à preuve du contraire, pourrait passer pour une inconséquence magistrale - eu égard aux projets initiaux - incombe à l'une des deux parties en présence, aux deux en même temps, ou encore si cette occasion manquée n'est pas le fruit d'autres facteurs plus généraux. En effet, aussi rapide soit-il, notre parcours ne peut manquer de prendre en considération l'ensemble des recherches sur les usages de la télématique. Or, jusqu'à maintenant, il a surtout été question d'un type relativement particulier de travaux ; ceux portant sur les incidences à moyen et long terme de la télématique du point de vue du développement et de la pertinence de ses usages sociaux.

Peut-on cependant établir le même diagnostic concernant l'autre volet de la recherche, qui a d'ailleurs été davantage sollicité par les promoteurs industriels et commerciaux, et pour cause puisqu'il s'agit de celui des expertises ? Avec ces productions d'experts, nous entrons dans un type tout différent d'études, moins attachées - pour reprendre notre distinction précédente entre usages et utilisations - à la dimension réflexive sur les usages mais plus directement destinées à résoudre des problèmes immédiats et concrets concernant les utilisations, dans des perspectives plus proches de l'ergonomie ou du marketing que de la sociologie.

29 La question du statut de ces travaux est d'importance, car s'il apparait que seules les recherches de la première catégorie ont été victimes, que ce soit ou non de leur faute, de l'incommunication qui vient d'être signalée, on pourra mettre cette situation sur le 
compte des difficultés que, traditionnellement, les recherches fondamentales - $a$ fortiori critiques - rencontrent au moment de la diffusion de leurs résultats. Dans cette hypothèse, le cas de la télématique ne serait finalement qu'une illustration de plus du clivage récurrent entre recherches à finalité appliquée et recherches à visée théorique, les premières étant naturellement parties prenantes des processus industriels, à l'inverse des secondes qui, par rapport à ces processus, adoptent explicitement une position d'extériorité, préférant prendre pour destinataire les instances politiques ou sociales.

Or, à nouveau, et avec toute la prudence qu'imposent de si brèves considérations, l'on doit également constater l'absence des experts au moment où on les aurait le plus attendus : lors des décisions, grandes et petites, qui ont marqué le développement technique, commercial et industriel du vidéotex.

31 Ce constat vaut pour la première phase, celle concernant la définition du système luimême. En témoigne, entre autres, l'erreur commise (par trois hauts fonctionnaires de la DGT) au moment du choix initial du type de clavier, abcd au lieu de l'azerty pourtant recommandé par les experts et auquel il a fallu revenir bien vite. En témoigne également l'improvisation qui a présidé à la conception du terminal, à la définition de la taille de l'écran et à la spécification des normes graphiques, malgré l'avis des experts et notamment de ceux, accrédités, de l'ODA, au sein de l'agence Havas.

Mais ce constat vaut aussi pour les conditions de la mise en oeuvre du programme. Ainsi le projet caressé, au départ, par les collaborateurs du Directeur Général des Télécommunications, Gérard Théry, et qui a bien failli connaître un début d'exécution, trahit-il leur méconnaissance des réalités sociales élémentaires: il s'agissait ni plus ni moins de contraindre tous les abonnés du téléphone à abandonner l'annuaire papier en échange du Minitel. Sans doute, dans ces conditions, n'était-il pas impensable en 1980 d'atteindre un parc de 30 millions de Minitel en 1990 ! De même les hésitations des PTT sur le financement du plan télématique - avec, en particulier, l'appel inopiné et sans lendemain aux ressources des collectivités locales - sont révélatrices de l'imprécision des données et de l'insuffisance de la réflexion financière préalable. A chaque fois, l'administration des PTT donne l'impression d'agir au coup par coup.

Quant aux fournisseurs de programmes - et tout particulièrement les fournisseurs des programmes administratifs, parmi les premiers à faire appel au vidéotex -, le moins que l'on puisse observer est qu'eux aussi ont avancé à l'aveuglette : généralement sans aucune idée de la nature et des caractéristiques psychosociologiques des utilisateurs potentiels, sans règle systématique pour l'agencement des informations et l'organisation des dialogues, à plus forte raison sans indication sur les coûts et les contraintes, ils n'ont pas trouvé de véritable secours auprès des organismes censés les conseiller, comme le CESI, le CESTA ou l'ADI, eux-mêmes de toutes façons dépourvus des moyens d'engager de véritables recherches.

Les entreprises de presse, pour leur part, n'étaient guère mieux armées et elles n'ont pas davantage tiré parti d'instances de réflexion et d'expertise pour l'évaluation des expériences réalisées et la connaissance du fonctionnement socioéconomique de la télématique. Cette situation est d'autant plus frappante que, parallèlement, sur les aspects exclusivement techniques, les ingénieurs de l'informatique et des télécommunications impliqués dans le développement des systèmes télématiques ont créé des structures telles que l'APST (devenue ensuite l'AFTEL), où les résultats sont, au fur et à mesure, activement examinés et diffusés auprès des milieux concernés. 

malgré tout, qui comptèrent pour beaucoup dans l'évolution favorable du plan télématique : le recours à Transpac et la fonction « Kiosque " par exemple. Mais elles témoignent davantage de la pertinence des intuitions des promoteurs que de la rigueur de leurs travaux préparatoires. D'ailleurs, la panne de Transpac, en juin 1985, due à un mauvais dimensionnement du réseau suffirait - si c'était nécessaire - à mettre en évidence qu'en matière de trafic les intuitions ne sont pas suffisantes. Quant aux prestataires de programmes, les succès de ceux qui survivent aujourd'hui ne doivent pas faire oublier les déboires que tous connurent au départ faute d'une appréhension des réalités et exigences socioéconomiques du nouveau médium. Nombre des pionniers n'eurent d'ailleurs pas les reins assez solides pour s'en relever...

\section{Histoires parallèles.}

Tout se passe donc comme si, aussi surprenant que cela puisse paraitre, deux histoires s'étaient déroulées en parallèle sans guère se rencontrer, serait-ce à l'occasion de la formulation d'un cahier des charges ou de la remise d'un rapport. D'un côté, la recherche sur les usages de la télématique, avec des finalités, des méthodes et des objets différents selon les chercheurs qui y participent. De l'autre côté, les structures de concertation et de décision politiques aussi bien que sociales et industrielles. Entre les deux, aussi peu d'interaction que possible. Au contraire, non seulement le fait que les premières dépendent, de près ou de loin selon les cas, des secondes ne semble pas avoir été un obstacle à leur imperméabilité mutuelle, mais encore c'est plutôt cette imperméabilité qui pourrait avoir facilité leur développement respectif.

sers, une explication, quelque peu inattendue, tend à se faire jour ici. Elle reprend en les complétant les deux explications précédentes sur la fuite en avant et sur la priorité des commandites publiques. Non seulement elle vise en effet à rendre compte de la multiplication initiale des productions scientifiques mais encore elle cherche à faire comprendre leur stagnation actuelle. En somme, elle suggère que les recherches auraient prospéré grâce à des instances décisionnelles qui auraient elles-mêmes tiré une forme de légitimité de leur ouverture aux chercheurs. Telle serait la circularité (presque) parfaite d'un scénario auquel, plus ou moins volontairement ou plus ou moins consciemment, les uns et les autres se seraient prêtés. Plus ou moins de bonne foi sans doute et en tout cas jusqu'au moment où, au cours de la seconde moitié des années 80 , l'écart se faisant trop grand entre recommandations et décisions, cette forme de gentlemen agreement devenait intenable. D'où le tarissement des études à partir de 1985.

\section{La fiction du microcosme.}

Encore faut-il, avant même d'essayer d'accréditer cette explication, qu'un premier aspect soit précisé, à propos de cette légitimité que la DOT aurait cherché à tirer en procédant à la consultation des chercheurs, et plus particulièrement des chercheurs universitaires dont la présence (relativement exceptionnelle) a été signalée dès le départ.

L'on n'a vraisemblablement pas assez remarqué en effet qu'en sollicitant les services de telles équipes universitaires (ou du CNRS), et plus seulement la contribution de bureaux d'études, comme c'était pratique courante précédemment, les décideurs de 
l'administration des télécommunications - ou tout du moins certains d'entre eux - ont fait plus que s'entourer des précautions habituelles d'une technocratie éclairée. La question se pose de savoir si, en réalité, leur démarche n'est pas au moins autant politique que scientifique. En faisant délibérément appel à ces équipes, composées de chercheurs dont les orientations critiques sont connues - et qui ont déjà eu l'occasion de les manifester publiquement par le passé, sur les problèmes de communication ou sur d'autres problèmes (tels que le nucléaire) - il est probable que ces décideurs ont sans doute entrepris en effet de s'adresser à des interlocuteurs qui, peu ou prou, sont aussi - ou, en tout cas, se veulent et se présentent comme - les porte parole des usagers. Ne sont-ils pas aussi garants de l'utilité sociale ? Aussi ne serait-ce pas malgré leurs orientations critiques qu'ils auraient été choisis, mais plutôt à cause de ces orientations.

Certes, l'on doit immédiatement préciser que la démarche d'ouverture en question n'a rien de spectaculaire, ni, en fin de compte, de trop périlleux pour les PTT. C'est essentiellement le département «Usages Sociaux des Télécommunications » du CNET, dirigé par Patrice Flichy justement, qui, seul ou en association avec le Service de la Prospective et des Études Socio-Économiques (SPES) de la DOT, a procédé à ces types d'appels d'offre. Or, ce département, de création récente (mais dont la création témoigne justement de l'orientation nouvelle des PTT), occupe lui-même - et, à certains égards, revendique - une position marginale. Cette position l'est tellement d'ailleurs qu'il arrive aux responsables de ce département de devoir rechercher des alliés et des cocommanditaires en dehors des PTT : au ministère de la recherche, à l'INA ou à la DGRST. En revanche, au sein des PTT, à côté de lui et parfois contre lui, d'autres structures telles que la Direction des Programmes et des Affaires Financières (DPAF) et surtout la Direction du Programme Télétel, au sein de la Direction des Affaires Commerciales et Télématiques (DACT), continuent à mener une politique de commandite uniquement tournée vers les études appliquées.

41 Peut-être est-ce toutefois justement cette marginalité du département « UST » qui le rend mieux à même d'entreprendre cette ouverture vers les chercheurs universitaires. Ce faisant, il ne contribue pas peu à l'établissement de cette sorte de fiction d'un microcosme où décideurs et chercheurs, associés dans la croyance que le niveau des usages de la télématique est un niveau pertinent d'analyse et d'action, cherchent à être davantage que les commanditaires et les contractants d'une série de programmes d'évaluation. En fait, leur interaction est censée représenter et, pour ainsi dire, mettre en scène en petit le jeu de toutes les forces et de tous les intérêts en présence : ceux de l'offre industrielle et ceux de la demande sociale.

Sans doute ne s'agit-il là que d'une fiction, mais entre ces différents acteurs - qui se connaissent parfaitement, sont issus des mêmes milieux scientifiques et se réclament peu ou prou de valeurs semblables - elle est assez forte pour sceller en effet une alliance dont ensuite le ministre des PTT lui-même pourra se réclamer afin de montrer combien son administration a rompu avec les pratiques technocratiques qui étaient en usage avant lui... Le problème, toutefois, tient à ce que si le département "UST " jouit d'une marginalité qui lui permet de procéder à cette ouverture, cette même marginalité se retourne contre son action et a fortiori contre la diffusion des recherches ainsi commanditées. Nous retrouvons ici notre problème initial. Celui de cette effervescence, facilitée ou encouragée par l'ouverture qui vient d'être indiquée, et stoppée presque dans le même mouvement. 

qu'au départ ou en cours de route, un incident se soit produit, qui ait empêché les deux histoires de se rencontrer. Faute de quoi on ne comprendrait pas comment, l'accord initial ayant été conclu, il n'a pas produit les résultats escomptés. D'où l'intérêt de nous reporter maintenant aux discours a posteriori en provenance des acteurs eux-mêmes, où la mention de cet incident figure bel et bien, même si, comme on pouvait s'y attendre, ce n'est pas le même qui est invoqué de part et d'autre.

\section{Reconstitutions croisées I : I'hypothèse des dés pipés.}

Commençons donc cet examen critique des tentatives de reconstitution par celle qui, émanant des chercheurs, tend tout naturellement à mettre les commanditaires en accusation. Ainsi Charon et Cherki (1984, p. 57), parmi les plus prolifiques de ceux qui ont été impliqués dans l'évaluation de Télétel, commencent-ils par mettre au crédit des promoteurs de la télématique le lancement d'une phase probatoire, mais pour faire immédiatement remarquer que "l'expérimentation elle-même, contrairement aux attentes, n'a suscité que fort peu de discussions et de réflexions de fond ».

Ce qui est intéressant dans l'explication qu'ils proposent alors, c'est qu'on y retrouve bien la référence à un scénario en deux temps : le premier où les contractants s'entendent sur l'objectif d'une expérimentation sociale ; le second, peu de temps après, où - " contre toute attente " - se produit la rupture implicite du contrat. Plus fondamentalement, toutefois, à l'origine de cette rupture, nos deux auteurs identifient deux facteurs intervenus successivement. D'une part, ils notent la hâte de la DGT dont ils incriminent le refus, jusqu'en 1982, qu' « on "cuise et recuise" les questions pour s'engager dans la diffusion massive du vidéotex »(p. 59). Après 1982, d'autre part, ils stigmatisent l'apparition d'« une nouvelle idéologie de la mutation technique et de la nouvelle culture à produire [...] devenue le "credo" des décideurs et l'objet d'un consensus social très large " (pp. 59-60). En d'autres termes, durant toute la période où le plan Télétel vit sous l'impulsion initiale, ce serait la DGT seule qui serait en cause ; ensuite - c'est-à-dire au moment où l'influence du gouvernement socialiste se fait sentir - l'ensemble des décideurs et l'opinion publique en général porteraient la responsabilité de la rupture.

L'un des arguments sur lesquels s'appuie cette reconstitution en forme de plaidoyer pro domo est celui, radical, selon lequel l'option télématique aurait été toute prête dès 1979, rendant d'emblée inutile tout questionnement sur les conditions et incidences du médium et de ses usages. Si la DGT a néanmoins fait appel à l'évaluation, c'était, selon nos deux chercheurs, pour la caution scientifique qu'elle représentait, manière de neutraliser à l'avance les critiques éventuelles sur le technocratisme, de contribuer à préparer l'opinion par toute une valorisation symbolique autour du Minitel et de donner par l'expertise ainsi sollicitée sa légitimité à la politique industrielle, Cela n'empêche, selon eux, que les dés étaient pipés dès le départ.

Avant de discuter du bien fondé de cette première interprétation, venons-en à la seconde, elle aussi tentative de reconstitution après coup, mais exactement symétrique de la précédente, car émanant principalement des milieux de la DOT. 


\section{Reconstitutions croisées II : le référent introuvable.}

Ainsi J. Dondoux, successeur de O. Théry, s'en prenant avec virulence à J .M. Charon, lors du colloque organisé par le CNET, dénonce-t-il le manque de fiabilité des données et résultats fournis par les chercheurs. Ce seraient donc eux - et non l'administration commanditaire des recherches - qui, selon lui, porteraient la responsabilité « morale " (selon ses propres termes) de la rupture du contrat. Ce seraient eux en effet - les chercheurs aux orientations critiques dont il a été question précédemment mais aussi l'ensemble des experts sollicités - qui auraient, notamment à Vélizy, méconnu la réalité du vidéotex, de ses usages et des attentes qu'il suscitait. A tel point, ajoute J. Dondoux, que si leurs diagnostics avaient été suivis, ils auraient amené les PTT à commettre l'erreur d'abandonner leur projet.

Derrière la polémique pure et simple, d'où des considérations liées aux querelles intestines entre la DOT et le CNET ne sont pas tout à fait absentes, il faut voir que les arguments de J. Dondoux ont un double intérêt. D'une part, ils reflètent assez bien l'opinion - et la déception sincère - de certains promoteurs de la télématique à l'égard des tentatives d'ouverture vers la recherche en sciences sociales. D'autre part, ils comportent également des éléments qui sont indispensables à la compréhension de ce qui s'est passé. C'est pourquoi même si le plaidoyer des commanditaires n'a pas la forme élaborée de celui des commandités, il est quand même intéressant, au travers des discours et de certains documents internes, d'y repérer l'argumentaire sous-jacent.

C'est ainsi que l'une des raisons de cette méconnaissance - dénoncée par J. Dondoux tiendrait à ce que, notamment dans le cas de Vélizy, l'investigation aurait été doublement faussée dès le départ : d'abord par l'établissement d'un protocole expérimental trop contraignant ; ensuite, plus grave encore, par la mise en question de ce même protocole. D'un côté comme de l'autre, la recherche était privée de l'une des conditions sine qua non de son bon fonctionnement : un référent fiable auquel rapporter les analyses.

51 Au début, en effet, un protocole strict, sur la base d'une technologie aux fonctionnalités spécifiées avec précision et aux utilisations prescrites par un mode d'emploi pré-établi, avait conduit le groupe de pilotage à identifier, sur les indications de la DGT elle-même, une population représentative. Le dispositif envisagé s'inspirait donc d'un modèle expérimentaliste où variables indépendantes (la technologie) et dépendantes (les réactions des usagers) étaient isolées et devaient être soigneusement quantifiées grâce à un échantillon. Non seulement, précisons-le, c'est la DGT qui a poussé à la mise au point d'une investigation de ce type mais encore, dans son principe, le modèle qui y préside ne fait que reprendre le plus simple des schémas « marketing » : sont analysées les réactions d'un utilisateur mis en présence d'une offre technique nouvelle.

Déjà, ce premier protocole - suivi de tous ceux qui procèdent de la même manière - révèle vite ses limites : adoptant pour référent un état donné de la technologie et de ses usages, il fige les situations en interdisant que soit prise en compte toute autre utilisation de la technologie et notamment toute utilisation déviante. Dès lors, ce qu'implicitement J. Dondoux lui reproche, c'est d'évaluer la télématique comme si elle était une technologie stabilisée, alors qu'au contraire elle est encore - et pour longtemps - à la recherche complémentaire de ses utilisations et de ses spécifications. Aussi l'investigation se rend elle-même a priori incapable d'enregistrer et de prévoir les évolutions à venir. Et J. Dondoux d'ironiser, comme l'ont fait bien des responsables de la DGT, en montrant 
comment les études sur la télématique ont, sauf exception, longtemps ignoré purement et simplement, puis fortement sous-estimé, même après ses premiers pas, la place que la messagerie allait occuper au sein des usages de la télématique.

Très rapidement d'ailleurs, dans le cas Télétel $3 \mathrm{~V}$, les circonstances viennent démontrer l'impossibilité de s'en tenir aux termes initiaux de l'analyse prescrite. Divers facteurs plus ou moins liés à la conduite même de l'expérimentation tels que l'insuffisance du nombre des foyers formant l'échantillon ainsi que des considérations de politique locale contraignent les expérimentateurs à élargir la zone test aux communes voisines de Vélizy, et ce avant que l'expérimentation ait débuté. Inévitablement, cet élargissement s'effectue au détriment des critères préalablement adoptés au point que c'est l'ensemble du plan expérimental qui s'en trouve déstabilisé. Cette situation nouvelle n'empêche pourtant pas l'Équipe «Projet » de Vélizy (Ponjrert et al. 1983) de s'en tenir à sa méthode expérimentaliste, même si celle-ci est désormais en porte à faux par rapport à la réalité observée et aux conditions de son observation. Comme l'écrit fort justement F. Séguy (1990b, p. 6) : «Avant même qu'elle ne commence, l'expérimentation de Télétel se base sur un dispositif expérimental déviant qui, simultanément, remet en cause sa nature expérimentale, mais lui permet d'avoir lieu ».

Sensible à la contradiction, J. Dondoux, amalgamant pour les besoins de la cause tous les types de recherche, y voit l'une des causes de l'auto-paralysie de la recherche évaluative en général : ou bien, suggère-t-il, celle-ci s'en tient à un schéma d'investigation fixé au départ, mais la réalité fluctuante des usages sociaux en cours d'élaboration lui échappe nécessairement. Ou bien, elle répudie tout schéma d'investigation, mais la recherche s'interdit alors toute appréciation objective. Évidemment l'alternative ne laisse pas de paraitre quelque peu caricaturale - et d'autant plus que, dans les limites de cette analyse, nous n'entrons guère nous-mêmes dans les détails. A dessein J. Dondoux omet les recommandations souvent pertinentes que les chercheurs lui ont adressées et dont quelques-unes ont été mentionnées précédemment : il n'est pire sourd que celui qui ne veut pas entendre! Toutefois, sur le fond, l'objection subsiste.

Sans doute la déstabilisation que nous venons de voir est-elle principalement d'origine circonstancielle, et les contradictions qui en ont résulté n'ont affecté, en l'occurrence, que les défenseurs et artisans d'une pratique expérimentaliste pure et dure de l'expérimentation sociale. Force est cependant de reconnaître que la mise en cause permanente des conditions présidant à l'implantation de la télématique a handicapé, en fait, l'ensemble des recherches sur les usages. Ainsi les transformations du terminal, la présence ou l'absence (selon les projets) de médiateurs chargés d'interroger les banques de données pour le compte des usagers finaux, le nombre croissant des services et la diversification des types d'utilisations, les modifications successives de la tarification et de la réglementation ainsi que bien d'autres changements intervenus tout au long de la phase de maturation du médium sont-ils quelques-uns de ces éléments déstabilisateurs qui ont régulièrement bouleversé en cours de route les données fondamentales de l'offre télématique, tout en étant eux-mêmes assez souvent les conséquences des initiatives des usagers finaux.

Or, même si les approches récusant les méthodologies expérimentalistes, inappropriées pour l'observation de situations fluctuantes, ont précisément pour caractéristique de chercher à développer au contraire des modalités souples d'observation, préférant le qualitatif au quantitatif, sans référent trop strict au départ, visant à enregistrer plus qu'à occulter les mutations susceptibles d'intervenir en cours de route, il n'en reste pas moins 
qu'ainsi soumises à de si importantes transformations - qu'en ces circonstances, elles enregistrent plus qu'elles ne les prédisent -, elles se trouvent inévitablement privées d'une grande part de leur marge de manoeuvre. Ainsi que le souligne alors J.L. Le Moigne (1982, p. 61), « l'expérimentation sociale perd son droit à dire le vrai, aussi onéreuse soitelle... ».

Que reste-t-il alors à l'expérimentation sociale ? Coincée entre, d'un côté, un référent trop contraignant et, de l'autre côté, une absence de référent, elle renonce en effet au vrai pour se contenter de dire ce qui est, au moment où cela est. Et en cela elle fait sans doute déjà beaucoup, même si, aux yeux d'un promoteur industriel et commercial, c'est nécessairement trop peu...

\section{Rendez-vous impossible.}

Malgré leur divergence, les deux interprétations - qui ne sont pas des témoignages stricto sensu mais valent ce que valent des reconstitutions après coup, sur la base de propos tenus à des fins de polémique et d'auto-justification - mettent l'accent sur un même aspect, fondamental, sur lequel, aussi curieux que cela puisse paraître, elles sont finalement d'accord. Ce qu'elles suggèrent en effet, c'est que les raisons de cette occasion manquée sont en réalité moins dues à des facteurs circonstanciels - la mauvaise foi et le cynisme des promoteurs industriels et commerciaux, l'incapacité des évaluateurs - qu'à des éléments structurels qui auraient en quelque sorte à la fois paralysé chercheurs et commanditaires. La question qui se pose maintenant est de savoir quels sont ces éléments structurels qui ont contribué à rendre impossible le rendez-vous.

Notre hypothèse est que ces éléments ne peuvent se comprendre que rapportés à une propriété constitutive des politiques présidant au lancement de la télématique, et peutêtre pas uniquement de la télématique mais de la plupart des nouvelles technologies d'information et de communication. Cette propriété serait leur ambivalence fondamentale, faite d'un mélange contradictoire de volontarisme et d'incertitude : volontarisme industriel d'un projet à mettre en oeuvre de toutes façons, incertitude concernant les applications de la technologie ainsi promue. Telle serait l'ambivalence paradoxale de la politique de promotion de la télématique qu'indirectement et sans doute involontairement, commanditaires et chercheurs impliqués dans l'évaluation de la télématique viendraient ici nous révéler.

Élément structurel en effet que le volontarisme industriel de la DOT, oeuvrant à la réalisation du plan Télétel malgré tous les obstacles qui lui sont opposés. Ce volontarisme, J .M. Charon et E. Cherki (1984, p. 52) le mettent en évidence en énumérant les dispositions adoptées par le ministère des PTT dès novembre 1978. Délais de mise au point de l'infrastructure technologique, durée de la phase expérimentale, rythme et volume de la distribution des terminaux, spécification des usages et des contenus: tout, suggèrent-ils, était prévu sans qu'apparemment retards, bifurcations et a fortiori interruptions soient même envisagés. Certes, des contraintes financières ou politiques ont ensuite obligé la DOT à infléchir certaines de ses orientations, mais, sur le fond, rien n'a été modifié : les jeux étant faits à l'avance, l'expérimentation restait nécessaire, du point de vue de la DOT, pour assurer la visibilité du programme mais elle devenait ipso facto inutile et, à plus ou moins brève échéance, gênante. 
61 A l'appui de ces indications et en remontant plus haut dans la chronologie nous pourrions également rappeler qu'effectivement le programme télématique a été lancé et conduit pour des raisons de politique générale, en vue d'optimiser les investissements réalisés lors de la décennie précédente pour la modernisation du réseau téléphonique et en vue de soutenir ou relancer par la commande publique les industries de l'électronique et des télécommunications. Dans les conditions d'une planification à si grande échelle, l'usage que la DOT pouvait faire de la recherche sur les usages était a priori des plus limités, surtout si les premiers résultats obtenus lui étaient défavorables. Aussi n'a-t-elle guère insisté pour en obtenir d'autres. Comme l'écrit B. Miège (in Bertrand et al 1990, p. 122), non sans quelque ironie, l'une des explications de la diminution de ces travaux tient à ce que " leur commanditaire principal, France Télécom, est aujourd'hui mieux" éclairé" ». Mais, on le verra, cette explication n'est peut-être pas la seule. D'autres raisons vont également devoir être recherchées, de l'autre côté.

En attendant, notons-le au passage, cela ne veut toutefois pas dire qu'il n'aurait pas pu ou dû - être de la responsabilité d'autres structures, observatoires indépendants, fonctionnant sur des fonds publics mais sans relation au moins directe avec les promoteurs industriels et commerciaux de la télématique, de suivre au fur et à mesure l'évolution du médium. Simplement, la DGT était, à coup sûr, le dernier des organismes à être en mesure d'assurer cette responsabilité sociale. L'erreur - si l'on peut parler ici d'erreur - a simplement été de la lui confier.

Élément structurel également - et en sens inverse cette fois - que l'incertitude présidant à la formation du médium. Toute politique volontariste ne procède pas nécessairement en effet d'une visée entièrement définie au départ, quand bien même l'idée du projet industriel et ses grandes étapes font, eux, l'objet d'une programmation rigoureuse. Au contraire, le vidéotex est l'exemple par excellence d'une technologie que l'on pourrait dire « flottante » (à la fois terminal et norme) et que ses promoteurs, d'ailleurs, n'ont pas hésité à laisser flotter assez longtemps. D'où les réticences de J. Dondoux envers les évaluations consistant à figer plus ou moins artificiellement la situation des usages.

Certes, l'acteur industriel reste toujours, bien évidemment, désireux de transformer au plus vitie son innovation en marchandise. L'on peut donc parler de volontarisme formel. Mais comme il ignore dans quel sens cette transformation s'opèrera, il peut se produire qu'il laisse de lui-même une certaine latitude à ses partenaires (industriels des programmes notamment) ainsi qu'aux premiers utilisateurs. Au volontarisme formel s'oppose l'incertitude objective. Bien loin de faire la chasse aux usages non prévus initialement, il lui arrive alors, au contraire, d'en encourager l'émergence, au besoin en suscitant des contextes particuliers où, dans des conditions privilégiées, notamment sans réelles sanctions économiques l'offre et la demande peuvent en quelque sorte s'exercer librement.

Peut-être même les grands programmes industriels en matière de nouvelles technologies de l'information et de la communication sont-ils tous plus ou moins condamnés à se soumettre à cette obligation d'intégrer des moments d'incertitude où stratégie industrielle et innovation sociale doivent faire bon ménage. C'est en tout cas ce que suggèrent Miège et al. (1986, pp. 245-246) à propos de l'industrialisation en général de la communication : "Capacité de création ou d'innovation et savoir-faire industriel sont donc entrés dans une phase d'articulation étroite ». Cette articulation, cependant, est en soi problématique : elle suppose de laisser aux usages sociaux le temps de Se développer, à partir de technologies aussi peu déterminées que possible et au prix de la mise en 
question ou du détournement de leurs fonctionnalités initiales, selon l'exemple de la messagerie pirate de Gretel. Mais ce qui, de l'extérieur, pourrait passer pour une forme d'opposition, voire de résistance, aux stratégies industrielles prescrites n'est-il pas en fait que la manifestation de la capacité propre à ces mêmes stratégies de laisser se développer la créativité sociale pour la récupérer à leur profit?

\section{Le paradigme ébranlé : des usages aux comportements sociaux.}

66 A la suite de ces analyses, il apparaît désormais que ce n'est pas en fonction d'un plan cyniquement élaboré par les promoteurs industriels ou encore à la suite dont on ne sait quelle incapacité des évaluateurs que la recherche sur les usages a été mise entre parenthèses ; c'est plutôt du fait de cette contradiction dans les termes que représente l'indétermination fondamentale d'un projet industriel volontariste ; c'est aussi du fait de l'instabilité qui en découle au niveau des situations expérimentales : au départ favorable à la mise en oeuvre d'expérimentations comme autant de situations d'exception où toute contrainte matérielle, notamment financière, est suspendue au seul profit de l'innovation technologique, cette indétermination, dans le même mouvement, se retourne contre le principe de cette recherche. En d'autres termes, impossible de mener une évaluation des usages quand la technologie par rapport à laquelle ces usages doivent normalement s'exercer n'est pas elle-même stabilisée. A la rigueur, une observation de ces usages...

Et encore, si l'on veut prolonger notre réflexion, n'arrive-t-on pas à l'idée que cette observation même est à son tour compromise ? Comme on l'a vu précédemment, la notion d'usage repose en effet sur le paradigme dialogique - lui-même inspiré du principe de l'échange bilatéral de l'offre et de la demande - d'une relation idéale et plus ou moins équilibrée entre deux pôles : d'un côté un utilisateur, sujet individuel ou, plus souvent, sujet collectif, supposé être en soi porteur d'attentes ou de besoins plus ou moins précis à l'égard d'une technologie ; de l'autre côté, cette technologie (ou cet ensemble de technologies), présentée comme une réponse, plus ou moins adaptée aux attentes de l'utilisateur, et dont en conséquence il est censé chercher à faire usage, en plus ou moins grande conformité avec le mode d'emploi qui lui en est transmis.

Que se passe-t-il cependant à partir du moment où, entre ces deux pôles, l'équilibre se rompt ? Que se passe-t-il, en d'autres termes, quand la technologie apparaît comme non stabilisée et quand, face à cette technologie, plus virtuelle que réelle, l'utilisateur luimême met davantage en oeuvre des comportements - qui font éventuellement (mais pas nécessairement) intervenir cette technologie - que des usages (au sens strict) de cette technologie ? C'est, selon nous, la notion même d'usage qui risque de s'en trouver disqualifiée.

Il est à cet égard assez caractéristique que nombre des évaluateurs aient été sensibles à la fragilité de la notion d'usage. C'est le cas par exemple de N. Benqué et S. Gauthronet (1984, p. 33), dont le commentaire embarrassé à propos d'une expérience de télématique rurale porte, nous semble-t-il, la trace de la difficulté que nous venons de soulever, mais qu'ils expriment plus qu'ils ne la résolvent : «Le fonctionnement même de l'expérience met en relief [...] le côté secondaire du dispositif technique par rapport à la primauté des comportements sociaux qu'il génère ». Quelle " primauté ", demanderons-nous, si c'est le dispositif technique qui «génère» les comportements sociaux ? Bien qu'ici, remarquons-le 
au passage, la mention d'usages sociaux ait déjà fait place à celle de comportements sociaux, la référence méthodologique encore sous-jacente présente toujours, pour reprendre la formule de J. Caune et B. Miège (1982, p. 34), « le risque d'accorder une excessive priorité à l'innovation technique en la séparant, de l'ensemble des pratiques culturelles dont on sait qu'elles se modifient lentement sous l'effet des lois structurelles [...] ». Comme le dit B. Miège (1984, p. 90) à l'occasion de « Claire » : « Cette approche ne donne qu'une vue fort restrictive des usages et des pratiques. Elle dissimule tout ce qui, à l'occasion du lancement de CLAIRE, a "travaillé" la réalité sociale locale ».

70 Le problème- qui, sans doute, fait hésiter N. Benqué et S. Gauthronet - est toutefois qu'en se déportant vers cet " ensemble des pratiques culturelles ", renvoyant lui-même à des " lois structurelles » que vont matérialiser des logiques sociales, décrivant des régularités de fonctionnement ; comme l'explique notamment P. Pajon (1990, p. 1), l'évaluateur risque en fait d'abandonner tout à fait la perspective des usages sociaux. Sous prétexte d'éviter une "vue fort restrictive des usages et des pratiques", ce sont ni plus ni moins les usages qu'il risque de perdre de vue, sortant du même coup complètement du mandat qui lui avait été assigné. En témoigne à sa manière A. Vitalis (1984, p. 101), l'un des principaux responsables de l'évaluation du programme « Telem » de Nantes:

Dès lors que l'on considère la télématique comme un atout économique fondamental et comme une technologie dont l'avènement est inéluctable, la question de savoir si elle répond ou non à des besoins sociaux n'a plus grande pertinence.

Or, avec la disparition des besoins sociaux, c'est bel et bien aussi celle des usages sociaux qui se dessine.

Et Vitalis d'ajouter cependant, à propos de la technologie et non sans regretter que les choses se présentent ainsi :

La vraie question est alors de savoir comment peuvent être créées les conditions de son intégration sociale (ibidem).

A travers cette "vraie question », observons le changement qui vient de s'opérer: ce ne sont plus les usages d'une technologie qui sont ici concernés, mais une intégration sociale, c'est-à-dire des comportements sociaux en présence de cette technologie. Éventuellement, sur un registre politique, cette " vraie question » sera alors celle de la mise en place de ce que R. Eksl (1982, p. 52) appelle des "véritables mouvements d'utilisateurs ", susceptibles, en un second temps, de constituer " une véritable assise militante pour faire contrepoids à la primeur de l'offre des constructeurs et de l'Administration ». Sur un registre sociologique, il s'agira, à l'opposé de toute recherche prenant pour objet « l'impact de ces techniques sur les modes de vie » d'étudier

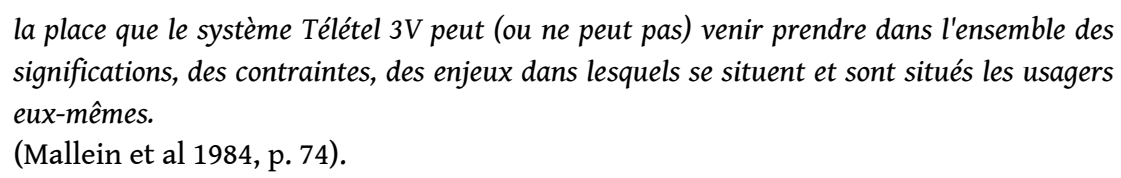

Autant de préoccupations qui éloignent les visées des évaluateurs de la pensée technicienne propre aux opérateurs industriels et commerciaux. Mais tout autant, et presque subrepticement, ces préoccupations renouvelées disqualifient pour l'évaluation la problématique en termes d'usage, en y substituant une autre problématique, en termes d'enjeux. 


\section{Migration silencieuse : un déficit d'esprit critique?} impliqués dans l'évaluation des usages sociaux de lálématique en soit venu progressivement et presque insensiblement à se détourner de cette question, exception faite pour le petit nombre de ceux qui continuent - mais pour des raisons très particulières - à trouver, notamment dans les dialogues télématiques, des terrains pour des analyses sociolinguistiques ? Assurément non puisque, comme on le voit plus clairement maintenant, c'est au moins autant par une conversion dont les causes sont à rechercher du côté des problèmes théoriques liés à la notion d'usage que par suite du changement de politique évaluative de la DOT que cette migration silencieuse s'est effectuée.

A partir de 1985-86, la majorité des chercheurs sur la télématique ont donc émigré, délaissant la question des usages pour s'attacher, en amont des situations et des pratiques concrètes de communication, à l'analyse des politiques publiques et des modes de coordination entre acteurs. Ce n'est pas que ces thèmes soient nouveaux. Simplement, il semble bien qu'ils constituent aujourd'hui la préoccupation dominante au sein de la recherche sociale en télématique, en lieu et place de la question précédente des usages sociaux qui, pourtant, on l'a vu, avait, un moment, structuré tout un champ de recherche. A cet égard, le numéro commun TIS et Réseaux est un bon indicateur de ce renversement de tendance : sur 7 articles, il n'en est plus qu'un, celui d'Arnal et Jouët (1989), encore consacré aux pratiques de la télématique. Les autres - avec, entre eux, de sensibles différences - touchent à des aspects concernant la généalogie des politiques industrielles, financières et commerciales et les stratégies organisationnelles.

Que penser d'une telle migration ? Sans être aussi sévère qu'A. Vitalis (Bertrand et al. 1990, p. 124) qui va jusqu'à déplorer le fait qu'« un déficit d'esprit critique caractérise l'ensemble des études réalisées sur la télématique », l'on peut, pour conclure, insister sur trois problèmes que pose la situation actuelle de la recherche en télématique.

- Premièrement, pour l'évaluation : la problématique des usages apparaît rétrospectivement comme le compromis d'une, période, celle de l'expérimentation sociale, où dominait le paradigme idéal de la construction dialogique du médium. Au nom de ce paradigme, la parité supposée entre la technologie et ses utilisateurs, les échanges, négociés à l'amiable Ou fruits de résistances acharnées, qui doivent s'établir entre ces deux pôles et, en fin de compte, le résultat de ces échanges, sédimentés sous la forme d'un ensemble plus ou moins constitué d'usages, étaient autant de principes tenus pour acquis : non seulement ils alimentaient toute une série d'études mais encore ils justifiaient, s'ajoutant à la sollicitation des habituels experts, la mobilisation de chercheurs que leurs orientations critiques, en d'autres circonstances, auraient tenus à l'écart de semblables concertations. Et de ces études, on attendait ni plus ni moins des indications pour infléchir les processus industriels dans le sens d'une meilleure adaptation aux besoins sociaux.

Commanditées de bonne foi et engagées avec conscience, ces études ne pouvaient cependant, dans la situation ambivalente de volontarisme et d'incertitude qui caractérise la promotion de la télématique, aboutir aux résultats espérés. Chronique d'un échec programmé : la mise en question de la notion d'usage déborde du strict cadre de la 
discussion théorique : elle en vient très rapidement à poser une autre question : celle de l'utilité de ces recherches et, par conséquent, de leur usage même.

Échec d'autant plus marquant que, toujours en retard d'une application ou déplaçant, malgré l'avis de leurs commanditaires, leur investigation des utilisations de la technologie aux comportements sociaux en présence de la technologie, ces études ont laissé un goût amer autant à ceux qui les ont réalisées qu'à ceux qui auraient dû les utiliser. Nul doute que ce rendez-vous manqué (et impossible) a sonné le glas du rapprochement timide qui s'esquissait à travers le mot d'ordre d'expérimentation sociale en ce début des années 80 , sur des actions évaluatives, entre promoteurs industriels et chercheurs en sciences sociales.

81 - Deuxièmement, si, à coup sûr, l'administration des PTT n'était pas - et de loin l'instance la mieux placée pour commanditer des travaux d'évaluation en matière de télématique, même en jouant de la marginalité relative de certaines de ses structures de commandite, cela ne signifie pas que ces travaux, avec le recul et à l'initiative d'observatoires ou d'organismes indépendants, ne restent pas indispensables.

De ce point de vue, une remarque de J .M. Charon et E. Cherki (1984, p. 60) à propos de Vélizy demeure valable aujourd'hui :

A partir du moment où il n' 'y a pas de bilan officiel, il ne peut pas non plus y avoir de débat sur les problèmes politiques, culturels, sociaux, économiques qui finalement se dégagent de l'expérience de Vélizy. Il ne reste plus alors qu'une multiplicité de "bilans », "leçons », " acquis »... non contrôlés de ce qui s'est passé.

Telle est bien encore la situation de la recherche en télématique. Plus que jamais, après dix années d'innovation, un bilan est nécessaire. En dépend le débat social sur la télématique, qui n'a toujours pas eu lieu.

84 - Troisièmement, la question se pose alors de savoir sur quelles bases organiser et réaliser un tel bilan. C'est ici, semble-t-il, qu'il conviendrait de tirer les leçons de la disqualification, officieuse mais réelle, qu'a connue la problématique en termes d'usages dans ses applications à l'évaluation de la télématique.

Cette disqualification, rappelons-le, ne veut évidemment pas dire que la notion d'usage n'aurait désormais plus d'utilité et que toute réflexion y faisant appel se trouverait du même coup disqualifiée. Ce qui est en question, ici, c'est l'exclusivité donnée à la notion d'usage, c'est-à-dire à une investigation se limitant au processus dialogique de construction en temps réel d'un médium. Comme si cette construction n'était pas déterminée par - et ne renvoyait pas à - des processus plus fondamentaux auxquels, justement, l'observation des usages demande à être référée : des stratégies industrielles, des déterminations idéologiques, des pratiques sociales, autant de paramètres qu'involontairement le fonctionnalisme des pratiques évaluatives risquait d'occulter.

Ce qu'en d'autres termes cette disqualification signifie, c'est que l'extension de problématique dont la perspective des usages témoignait déjà (par rapport à la perspective des utilisations) - et qui a rendu possible la mobilisation scientifique que nous avons vue - n'était, en fait, encore pas suffisante, ni d'un point de vue strictement théorique, ni, corrélativement, pour permettre aux chercheurs ainsi mobilisés d'arriver aux résultats attendus. C'est donc, dans le même mouvement, cette première extension qu'il s'agirait aujourd'hui de prolonger.

Prolongement d'autant plus nécessaire que l'on peut craindre en effet qu'avec le reflux de la recherche sur les usages, l'idéal même d'une perspective intégrée, à la fois globale et 
transversale, ne s'éloigne subrepticement. Faut-il donner, concernant la télématique, un exemple des raisons qui pourraient alimenter une telle crainte ? Ce sera celui de l'utilisation, pour le moins abusive, qui est presque universellement faite aujourd'hui, jusque et y compris dans les milieux de la recherche sociale (pourtant naguère encore circonspects), de la notion de réussite pour qualifier le développement de la télématique française.

A dire vrai, la notion de réussite ne signifie pas grand chose tant qu'elle n'est pas, en bonne méthode, assortie de la double mention, d'une part, de ce qui est considéré, d'autre part, de l'étalon à partir duquel cette réussite doit être mesurée. Or, faire apparaître ces deux mentions, c'est justement rompre avec la pratique, encore dominante, des points de vue séparés et s'engager en connaissance de cause dans une évaluation globale. Réussite technologique ? Réussite industrielle ? Il n'est pas sûr que l'ensemble Télétel (norme + terminal) l'emporte à cet égard sur ses rivaux, Télidon et Prestel qui, chacun dans leur genre et à leur époque, ont été des innovations plus significatives. Et si la diffusion du Minitel est une réussite par rapport à celle des terminaux à l'étranger, on ne précise pas assez que c'est sur la base d'une distribution gratuite, réalisée nulle par ailleurs, sans point de comparaison par conséquent. Quant à la réussite financière, le récent rapport de la Cour des Comptes introduit de sérieuses réserves sur la rentabilité de l'opération, pour les PTT en tout cas. De même, le volume général de la consommation demande, pour être caractérisé comme une réussite, à être rapporté à d'autres données, celles de l'évolution dans le temps par exemple, en référence notamment au modèle de la courbe logistique, pour l'utilisation de laquelle il faut bien reconnaitre que l'on ne dispose pas, pour le moment, d'un recul suffisant. Surtout, l'évocation séparée de toutes ces réussites (plus ou moins effectives au demeurant) montre bien au devant de quels dangers la recherche s'engage si elle perd le principe et l'idéal d'une évaluation intégrée, faisant jouer et mettant systématiquement en relation de manière transversale les différents niveaux et les différents facteurs les uns par rapport aux autres.

89 Ce n'est pas le moindre des mérites de l'expérience de la recherche sur les usages de la télématique que d'en témoigner à sa manière, même si, en fin de compte, cette expérience est une expérience malheureuse. De fait, elle en témoigne en positif, par le niveau supérieur d'intégration qu'a effectivement représenté la notion d'usage dans l'évolution des sciences de la communication. Elle en témoigne tout autant en négatif, par les limites du paradigme dialogique sur lequel cette notion reposait, limites qui sont d'autant mieux apparues que, parallèlement, l'usage même des recherches qui faisaient appel à la notion d'usage apparaissait problématique.

\section{BIBLIOGRAPHIE}

Amal, N. \& Jouët, J., (1989), « Télétel : images des utilisateurs résidentiels », TIS et Réseaux, novembre 1989, pp. 105-124.

Benqué, N. \& Gauthronet, S., (1984), « Télétel et l'information du public rural », Réseaux n 7, pp. 1-33. 
Bertrand, G., Miège, B. \& Vitalis, A., (1990), « Débat autour du numéro spécial : Dix ans de Vidéotex", TIS, vol. 2, n 3, pp. 116-126.

Caune, J. \& Miège, B., (1982), « Réflexions à propos de quelques expérimentations sociales sur des systèmes télématiques ", Bulletin de l'IDATE $n^{\circ} 9$, L'expérimentation sociale en télématique, acte des $4^{e}$ journées internationales, octobre 1982, pp. 34-36.

Charon, J .M. \& Cherki, E., (1984), « Vélizy ou les premiers pas de la télématique grand public », Réseaux $n^{\circ}$ 6, avril 1984, pp. 49-68.

Eksl, R., (1982), « Modernisme technique et conservatisme social : deux caractéristiques des expériences françaises de télématique ", Bulletin de l'IDATE n 9, L'expérimentation sociale en télématique, acte des $4^{e}$ journées internationales, octobre 1982, pp. 49-54.

Flichy, P., (1984), « Éditorial », Réseaux n 6, avril 1984, 1 page.

Jouët, J., (1989), « Une communauté télématique : les axiens », Réseaux n³8, décembre 1989, pp. 49-66.

Le Moigne, J.L., (1982), « Pourquoi expérimenter alors qu'il est si simple de réfléchir ? Pour une critique de la méthode expérimentale », Bulletin de l'IDATE $\mathrm{n}^{\circ} 9$, L'expérimentation sociale en $^{2}$ télématique, acte des $44^{e}$ journées internationales, octobre 1982, pp. 55-66.

Mallein, P., Toussaint, Y \& Bydlowski, M., (1984), « TÉLÉTEL 3V, Les adolescents et leur famille ", Réseaux n 6, avril 1984, pp. 69-84.

Miège, B., (1984), « Les enjeux du vidéotex interactif : réflexions sur l'expérimentation de Claire (Grenoble) », Réseaux n 6, avril 1984, pp. 89-92.

Miège, B., Pajon, P. \& Salaün, J.M., (1986), L'industrialisation de l'audiovisuel des programmes pour les nouveaux médias, Aubier, Res Babel, Paris.

Pajon, P., (1990). « Videotex as a cultural industry », Videotex lessons from early research, IAMCR 17th Conference, Bled, Yugoslavia, 26-31 August, 4 p.

Ponjrert, M., Georgiades, P. \& Magnier, A., (1983), Communiquer par Télétel. Les acquis des expériences Télétel $3 \mathrm{~V}$ et de l'annuaire électronique en Ile-et-Vilaine, La Documentation française, Paris.

Séguy, F., (1990b), « Les stratégies publiques d'introduction du vidéotex en RFA et en France », Études et Travaux du G.R.I.C.C. $\mathrm{n}^{\circ} 4,28$ pages.

TIS et Réseaux, (1989), Dix ans de vidéotex, novembre 1989.

Vitalis, A., (1984), «Les enjeux socio-politiques et culturels du système télématique TELEM », Réseaux nº, avril 1984, pp. 99-116.

\section{RÉSUMÉS}

La situation de la recherche sur les usages de la télématique est paradoxale. Le nombre d'études et la structuration théorique du champ sont autant de signes de son intense développement. Mais d'autre part, elle donne l'impression d'une inutilité à peu près complète, comme en témoigne l'influence quasi nulle que ses résultats ont eue non seulement sur les débats sociaux mais encore sur les décisions industrielles. L'on montre comment cette situation n'est pas à mettre sur le compte d'on ne sait quelle inconséquence des chercheurs eux-mêmes ou de leurs commanditaires. Elle serait plutôt le résultat des limites théoriques de la notion même d'usage. Appliquée à l'évaluation celle-ci n'apparaît pas en effet comme un outil adéquat pour analyser l'indétermination qui préside au développement parallèle de l'innovation technique et des 
comportements sociaux. Surtout, cette notion repose sur le paradigme, rapidement contesté, d'un médium dont la formation serait issue d'un échange équilibré et paritaire entre offre et demande.

The situation of research about the uses of telematics is a paradoxal one. On the one hand, the numerous studies and the theoretical structuration of this field are obvious signs of their dramatie increase. On the other hand, they convey the impression of their almost complete uselessness, as can be seen through their absence of effects upon public opinion as weIl as upon industrial policies. This paper shows that such a situation does not result from a failure of searchers or policy makers. Our hypothesis is that coud be the consequence of the lack of definition of so called « uses ». In fact, in the context of evaluation this notion of « uses " has no practical efficiency in analysing the parallel developments of technical innovation and social practices. The point is that notion is based on the paradigm of a medium formed by the symetric interaction between offer and demand, which paradigm appeared very soon as a non convenient one.

\section{INDEX}

Keywords : telematics, usage studies, videotex, epistemology, politics of research, public policy Mots-clés : télématique, étude d'usages, vidéotex, épistémologie, politique de la recherche, politique publique

\section{AUTEUR}

PIERRE MOEGLIN

Pierre Moeglin, Université Paris Nord 